\title{
Designing ordered DNA-linked nanoparticle assemblies
}

\author{
D B Lukatsky' ${ }^{1}$ B M Mulder and D Frenkel \\ FOM Institute for Atomic and Molecular Physics [AMOLF], Kruislaan 407, 1098 SJ Amsterdam, \\ The Netherlands \\ E-mail: lukatsky@fas.harvard.edu
}

Received 10 July 2005 , in final form 2 November 2005

Published 19 April 2006

Online at stacks.iop.org/JPhysCM/18/S567

\begin{abstract}
We present a model study of a multi-component system that can form lowsymmetry ordered phases, even though all pair interactions between the constituent particles are spherically symmetric. Using Monte Carlo simulations and a mean-field model we investigate the thermodynamic, structural, and kinetic aspects of the formation of stripe phases for a simple, multi-component lattice model. This lattice model was chosen to represent a mixture of spherically symmetric DNA-coated colloids with several species of DNA linkers. We predict that the optimal strategy to design a specific low-symmetry phase is the one which not only provides the weakest strength of competitive binding, but also leads to the 'staged' ordering of nanoparticle superstructures.

(Some figures in this article are in colour only in the electronic version)
\end{abstract}

\section{Introduction}

A key goal in the development of materials with nano-sized building blocks is the ability to "design" ordered phases. To achieve this, the properties of the building blocks should be chosen such that the system will self-assemble into a prescribed structure [1,2]. One promising strategy is to use nanoparticles (spherical or non-spherical) that can be linked specifically and selectively. The molecules that are responsible for this specific interaction are termed linkers. One example is DNA-linked nanopaticle assemblies, where the particles are covered with single-stranded DNA (ssDNA) molecules of specific sequences [3-12]. This system was first introduced experimentally by Mirkin and co-workers [3]. These DNA-coated nanoparticles can be selectively linked by complementary ssDNA linkers. For every pair of nanoparticles there can be specific linkers that bind selectively to this pair, figure 1. The size of the colloidal particles involved varies from tens of nanometres in $[3-5,9-11]$ to a micron in $[6-8,12]$.

1 Present address: Department of Chemistry and Chemical Biology, Harvard University, Cambridge, MA 02138, USA.

0953.8984/06/180567+14\$30.00 2006 IOP Publishing Ltd Printed in the UK 


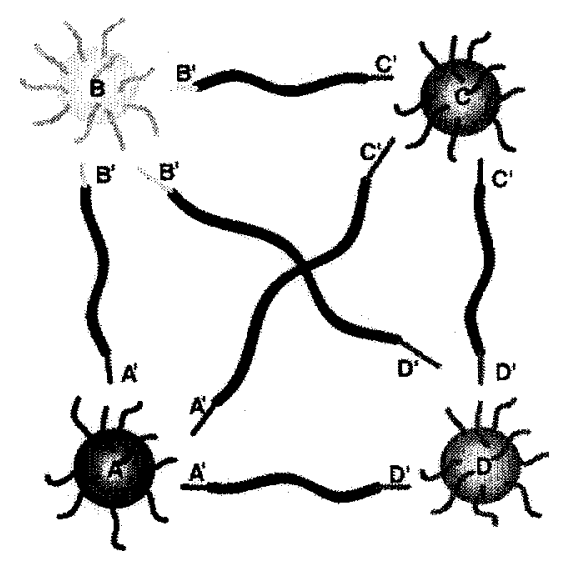

Figure 1. Schematic representation of SSDNA-coated colloids and complementary linkers. In the fout-component system of colloids of types A, B, C, and D, each colloid species is grafted with ssDNA molecules of specific sequence. These ssDNA sequences uniquely define the colloid fdentity. Complementary linkers e.g. $\mathrm{A}^{\prime} \mathrm{B}^{\prime}$ bind selectively and specifically the corresponding colloid pairs, in this case colloids A and B, respectively. Linkers are composed of the recognition SSDNA ends complementary to the corresponding 'probe' colloid pair, and a spacer (coloured in black). Spacers can be either dSDNA molecules, or can be buit using a nanomaterial, such as e.g. carbon nanotubes [18], or possibly microtubules, or actin molecules. In recent experiments the colloid size varied from tens of nanometres 5,9$]$ (with about a hundred SSDNA molecules grafted onto each colloid) to a ricron [6] (with thousands of SSDNA molecules on each colloid). We predict that using linkers of different lengths which provide inter-colloid bonds of different spacings one can design low-symmetry structures.

All these experiments used relatively short ssDNA chains grafted onto the colloids and short ssDNA linkers, of the order of a few nanometres (up to a few tens of nucleotides long).

The existence of such multi-component systems with 'programmable' pair interactions opens the way to the self-assembly of complex, functional nanostructures in both two dimensions (2D) and 3D. It was demonstrated recently that DNA-coated colloidal particles can indeed be programmed to self-assemble into a crystal [6]. Alternatively, but obviously far more laboriously, they can be assembled 'by hand' into a designed, ordered structure using a laser-tweezers set-up [7].

An important question is how to choose the properties of the elementary building blocks in order to make them self-assemble into a desired structure. In particular, we need to select interactions between the particles such that the desired structure forms spontaneously. This is a major issue, for example, in the design of self-assembling electronic circuits [19] or (photonic) crystals [20]. One approach to this problem is to design nanoparticles with functional 'patches', where the binding sites are located at specific positions on the surface of a particle [1, 16-18] (for a simulation study see e.g. [15]). Thus far, few practical realizations of these systems have been achieved due to the difficulty in manufacturing such 'patchy' particles (see e.g. $[1,16]$ ). Another approach is to use DNA scaffolds as templates for self-assembly of colloids [13, 14], but this strategy also requires the pre-assembled DNA templates themselves to be designed [13].

Clearly, it would be very attractive if non-spherically symmetric structures could be made to self-assemble out of spherically symmetric building blocks. The problem of the design of crystal structures consisting of particles with interactions that can be varied at will is somewhat similar to the problem of the design of protein structures. The objective in both cases is to make the system self-assemble into an ordered structure that is kinetically accessible. In some 
respects, the case of DNA-programmed self-assembly is easier than that of protein folding as there is no frustration imposed by the presence of a protein backbone. The key objective therefore is to choose the interaction parameters such that they facilitate the kinetics of selfassembly. The kinetics depends on the details of interaction between the particles. There is no general recipe that tells us how to combine thermodynamic and kinetic properties, even for one-component systems. However, Tkachenko et al have recently addressed this question for a restricted class of potentials modelling DNA-induced colloid interactions $[22,21]$.

Here, we consider a very simple model system to explore the relation between pair interactions, phase stability and kinetics of self-assembly. In particular, we consider the design of wire-like structures using spherically symmetric DNA-coated particles and a set of complementary linkers as building blocks. Stripe structures can be particularly important for applications, such as the construction of DNA nano-wires [13]. As DNA molecules can be metallized in a controlled way according to the sequence [19], the interconnection between functionally different nanoparticles can be established. For the sake of simplicity we shall consider a two-dimensional (2D) system of colloids with circular-symmetric interactions, which we map onto a lattice model. The circular symmetry on the lattice means that the interaction potential between any two nearest neighbour (or next-nearest neighbour) particles is independent of the orientation of this particle pair on the lattice. Our aim is to demonstrate the design principles of building low-symmetry structures using spherically (circular in 2D) symmetric building blocks. A quasi-2D DNA-linked colloidal system can be realized practically by adding an extra interaction of particles with the surface. This can be achieved by e.g. using one extra linker ssDNA species connecting specifically particles with the surface grafted with complementary, 'probe' ssDNA.

\section{Self-assembly of stripe structures}

In this section we first introduce the lattice model of the four-component system. Afterwards, we explain how one could change practically the range and strength of interactions in systems of DNA-coated colloids and DNA linker molecules.

\subsection{Lattice model of stripe-phase formation}

We consider a lattice gas model with particles of four types A, B, C and D which occupy lattice sites of a square, $2 \mathrm{D}$ lattice. For the sake of simplicity we assume that the concentrations of all particles are equal and fixed. The energy of this system is

$$
\frac{H}{\epsilon}=\sum_{\mathrm{nn}} J_{S_{i} S_{j}}+\sum_{\min } K_{S_{i} S_{j}},
$$

where the 'spin' variable $S_{i}$ takes on the value A, B, C, and D depending on which type of particle is occupying the site $i$, and we assume that all lattice sites are occupied i.e. we rule out vacancies. $J_{S_{i} S_{j}}$ and $K_{S_{i} s_{j}}$ are $4 \times 4$, symmetric, and dimensionless matrices of interactions for the nearest neighbour ( $\mathrm{mn}$ ) and next-nearest neighbour ( $\mathrm{mn}$ ) particles, respectively. $\epsilon$ is a positive constant setting the energy scale for the interaction. The summation in equation (1) is over all nn particle pairs $i$ and $j$ in the first sum, and non particle pairs in the second sum, respectively.

The ordered stripe structure that forms the 'design goal' of our system is shown in figure 2. The choice of the interactions between the particles intended to achieve this design goal as a stable state of the system is guided by the following logic. In order to favour the relative arrangement of the particles as shown in figure $2(\mathrm{a})$ we set $J_{\mathrm{AB}}=J_{\mathrm{BC}}=J_{\mathrm{CD}}=$ 


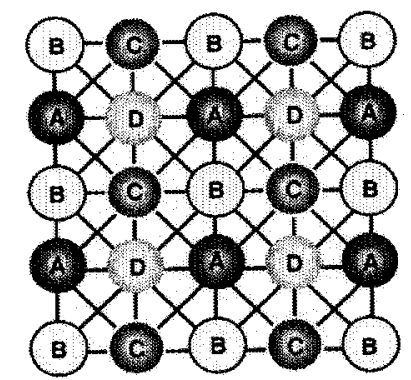

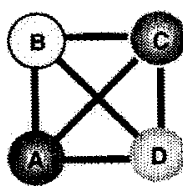

a

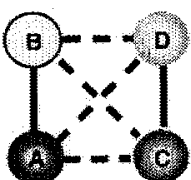

b

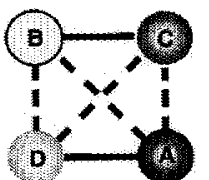

c

Tigure 2. Schematic representation of the ordered stripe structure composed of particles of four species with spherically symmetric interaction potentials. This structure represents the ground state of the system with the network of interactions shown in the botrom plots (a). The solid lines connecting the particles represent attractive interactions, and the dastied lines represent competitive interactions. In particalar, in order to tealize the stripe phase shown here, the strength of competitive attractions (dashed lines in (b) and (c)) should be weaker compared to the attraction between "native" nn and unn particle pairs.

$J_{\mathrm{AD}}=K_{\mathrm{AC}}=K_{\mathrm{BD}}=-1$. The specificity of the virtual linkers that gives rise to these interactions, imply that interactions between like type particles are disfavoured, and we set $J_{S S}=K_{S S}=+1$, for all species S. Finally, the remaining interactions, shown as dashed lines in figure 2 , which we collectively denote as competitive bindings, are given the strength $\delta \equiv J_{\mathrm{AC}}=J_{\mathrm{BD}}=K_{\mathrm{AB}}=K_{\mathrm{BC}}=K_{\mathrm{CD}}=K_{\mathrm{AD}}$, where $\delta$ is variable. We distinguish the regime of weak competitive binding when $\delta$ is positive and the system strongly prefers only the ground-state-like configurations, and strong competitive binding when $\delta$ is negative, which tends to destabilize the ground state and favours the configurations shown in figures $2(\mathrm{~b})$ and $(\mathrm{c})$.

We first investigate the thermodynamic and structural properties of the system. We use the specific heat per particle, $c$, characterizing energy fuctuations in the system as our probe for the thermodynamics. It is defined as

$$
c=\left\langle\left(\Delta H / k_{\mathrm{B}} T\right)^{2}\right\rangle / N,
$$

where $\Delta H=H-\langle H\rangle, N$ is the total number of paticles in the system, and the statistical averaging in equation (2) is performed with the Boltzmann weight proportional to $\exp \left(-H / k_{\mathrm{B}} T\right)$, where $T$ is the temperature and $k_{\mathrm{B}}$ is the Boltzmann constant.

We pertormed MC simulations on a lattice with particles of four different species, figure 2 . The computed specific heat per particle is shown in figure 3 . We observe qualitatively different behaviour of the specific heat as a function of $\delta$. In particular, in the case of weak competitive binding, figure $3(a)$, the transition to the stripe phase is very broad with two peaks in the heat capacity. These two peaks correspond to the hierarchical ('staged') rearrangements of the particle super-structures in the system. Similar anomalies in the heat capacity, corresponding to successive magnetic phase transitions, are experimentally observed in intercalation compounds [25]. 


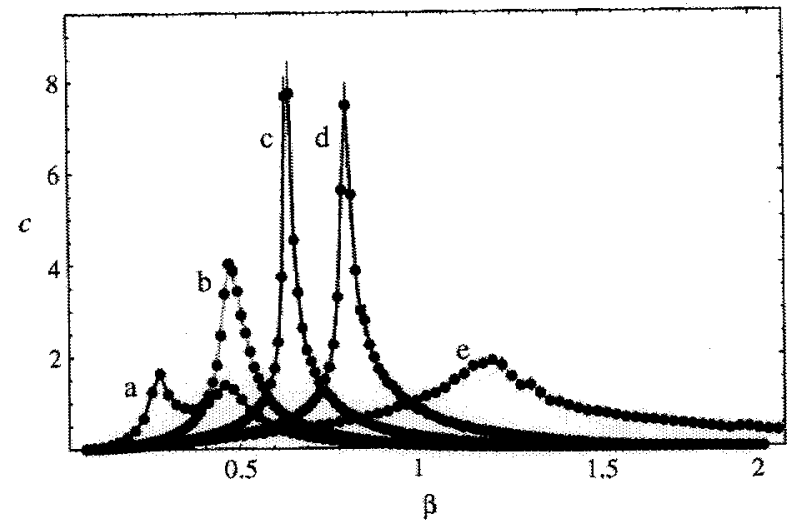

Figure 3. Specific heat per particle as a function of the inverse temperature $\beta=\epsilon / k_{3} T$ in the four-component system of A, B, C, and D particles (see figure 2). All five curves are computed with the same ground state energy, determined by the couplings $J_{\mathrm{AB}}=J_{\mathrm{BC}}=J_{\mathrm{CD}}=J_{\mathrm{AD}}=$ $K_{A C}=K_{\mathrm{BD}}=-1$, and $J_{\alpha \alpha x}=K_{\alpha \alpha \alpha}=+1$, for $\alpha=\mathrm{A}, \mathrm{B}, \mathrm{C}, \mathrm{D}$. The strength of competitive binding $\delta=J_{A C}=J_{\mathrm{BD}}=K_{\mathrm{AB}}=K_{\mathrm{BC}}=K_{\mathrm{CD}}=K_{\mathrm{AD}}$ is +1 in (a), 0 in $(\mathrm{b}),-0.5$ in $(\mathrm{c}),-0.75$ in (d), and -1 in (e). MC simulations were petformed with 1024 particles, and with open boundary conditions. In (e) the ground state is the columnar-like structure of stripes ordered in ID only.

With increasing strength of competitive binding (i.e. more negative $\delta$ ), the order-disorder transition first becomes sharper $(\delta=0, \delta=-0.5$, and $\delta=-0.75$ in figure 3 ). However, upon further decreasing of $\delta$, the peak of the heat capacity gets broader again, and reaches its broadest form at $\delta=-1$, figure 3 (curve (e)). This is a special case (with $\delta=-1$ ) where the system undergoes a transition to the columnar-like phase with stripes ordered in ID only.

In order to elucidate the structural signature of the behaviour of the specific heat we introduce the local, structural order parameter-the number of 'native' contacts between $\mathrm{nn}$ and nnn particles. In particular, we define

$$
\eta(\alpha \beta)=\frac{n_{\alpha \beta}}{n_{\alpha \beta}^{\max }},
$$

where $n_{\alpha \beta}$ is the number of nn colloid pairs $\alpha \beta$, and $n_{\alpha \beta}^{\text {sax }}$ is the maximal possible number of $\mathrm{nn}$ colloid pairs $\alpha \beta$ (in the ground state for a given lattice). Analogously, the order parameter is defined for nnn colloid pairs, $\eta^{\text {next }}(\alpha \beta)=m_{\alpha \beta} / m_{\alpha \beta}^{\text {max }}$, where $m_{\alpha \beta}$ is the number of nnn colloid pairs $\alpha \beta$, and $m_{\alpha \beta}^{\max }$ is the maximal possible number of nun colloid pairs $\alpha \beta$. For example, nn 'native' contacts for the structure shown in figure 2 are $\mathrm{AB}, \mathrm{BC}, \mathrm{CD}$, and $\mathrm{AD}$; and nnn 'native' contacts are $\mathrm{AC}$ and $\mathrm{BD}$.

We also define the fluctuation $\Delta_{\eta}$ of the order parameter $\eta$ :

$$
\Delta_{\eta_{i}}(\alpha \beta)=\frac{\left\langle\left(n_{\alpha \beta}-\left\langle n_{\alpha \beta}\right\rangle\right)^{2}\right\rangle}{n_{\alpha \beta}^{\max }},
$$

and analogously, the fiuctuation of the order parameter for nnn colloid pairs: $\Delta_{\eta}^{\text {sext }}(\alpha \beta)=$ $\left\langle\left(m_{\alpha \beta}-\left\langle m_{\alpha \beta}\right\rangle\right)^{2}\right\rangle / m_{\alpha \beta}^{\max }$. The statistical averaging in equation (4) is performed as the $\mathrm{MC}$ ensemble averaging.

We computed the average values of the order parameter $\langle\eta\rangle$ and the fluctuation of the order parameter $\Delta_{\eta}$ in two cases corresponding to the two different types of behaviour of the specific heat $c$, figures $3(\mathrm{a})$ and (c). In particular, figure 4 shows the case with $\delta=+1$, where $c$ has two peaks (figure $4(a)$ ). In figures $4(b)$ and (c) diamonds correspond to the on $A B$ colloid 


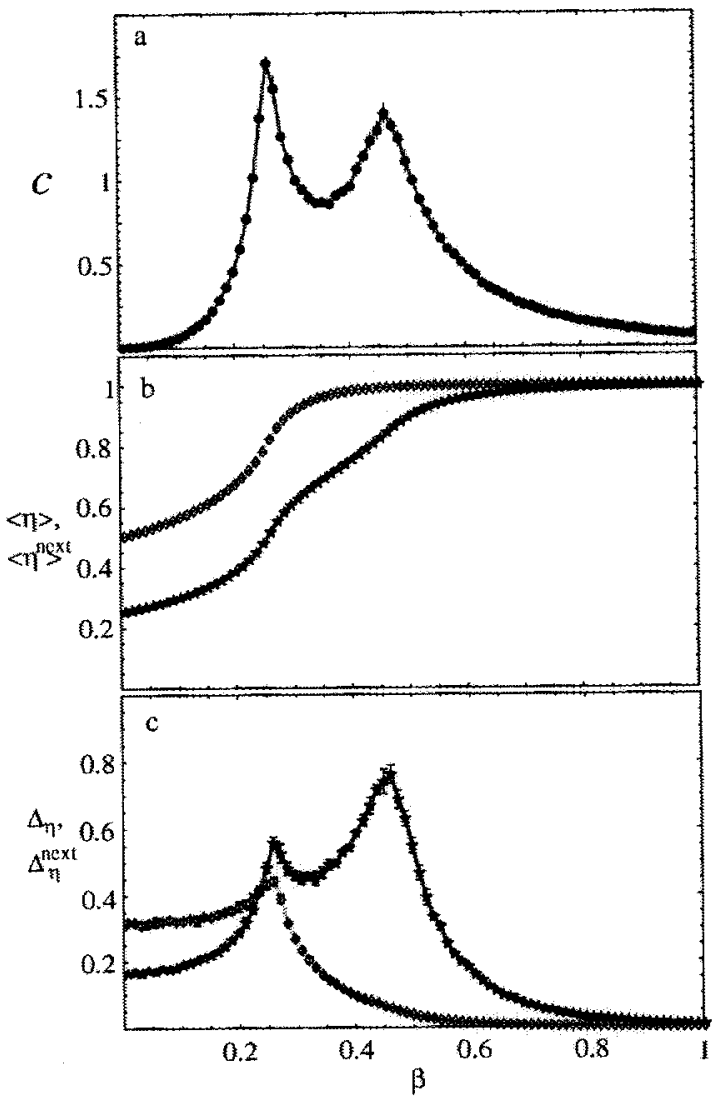

Figure 4. Computed themodynamic and strictural properties of the four-component model in the case of weak competitive binding: specific heat $c$ per particle (a); mean valuc of the order parameter $(\eta)$ in (b); and fuctuation of the order parameter $\Delta_{\eta}$ in (c). In (b) and (c) the two curves with diamonis and stars correspond to the nearest neighbour $\mathrm{AB}$ and the nex-thearest neighbour $\mathrm{AC}$ particle pairs, respectively (see main text and figure 2). The interaction network used to compute these plots is identical to the one used to compute figure $3(a)$, where $\delta=+1$. MC simulations were performed with 1024 particles and with open boundary conditions.

pairs, and stars correspond to mnn colloid AC pairs (see also schematic plot, figure 2). At low values of temperature (large $\beta \equiv \epsilon / k_{\mathrm{B}} T$; this $\beta$ should be distinguished from the subscript $\beta$ !) these order parameters saturate at one, as the system reaches the ground state (figure $4(b)$ ). The most prominent feature in figure 4 is that $\Delta_{\eta}$ for nn and nnn 'native' contacts exhibit two consecutive transitions at two different values of $\beta$. The behaviour of the order parameter is very different in the case of strong competitive binding, $\delta=-0.5$ (see figure 5 ). In this case the disorder-order transition is characterized by the cooperative ordering of both $\mathrm{nn}$ and ann colloid pairs (figure $5(\mathrm{c})$ ).

The first peak of the specific heat (figure 4(a)) corresponds to the ordering transition of the nn, favourable colloid pairs (we also term these m native contacts). This is evident from figure 4(c) (diamonds). In this particular case the nn native contacts are those formed by $\mathrm{AB}, \mathrm{BC}, \mathrm{CD}$, and $\mathrm{AD}$ nn colloid pairs. At the same time, there is also a pre-ordering of nnn native contacts (nnn $\mathrm{AC}$ and $\mathrm{BD}$ colloid pairs). This is represented by the first, weak peak in 


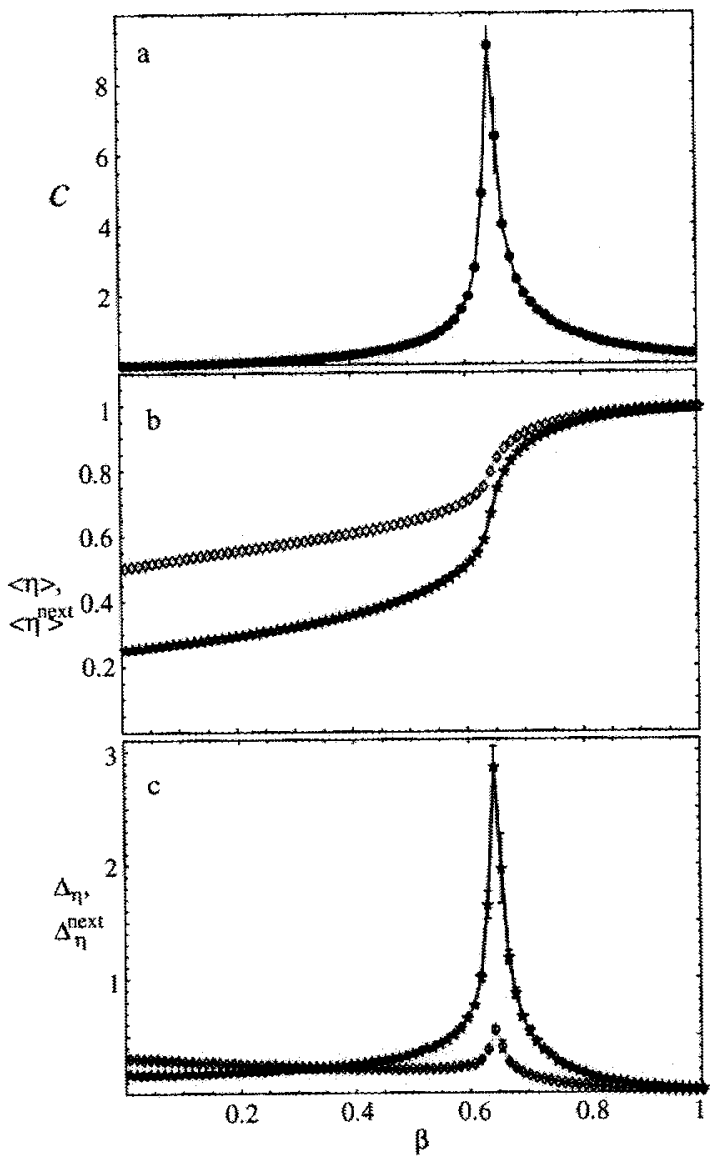

Figure 5. Computed thermodynamic and structural properties of the four-componert model in the case of strong competitive binding: specific heat $c$ per particle (a); mean value of the order parameter $\{n\rangle$ in (b); and luctuation of the order parameter $\Delta_{n}$ in (c). In (b) and (c) the two curves with diamonds and stars correspond to the nearest neighbour $A B$ and the next-nearest neighbour $A C$ particle pairs, respectively (see main text and figure 2). The interaction network used to compute these plots is dentical to the one used to compute figure $3(\mathrm{c})$, where $\delta=-0.5$. MC simulations were performed with 1024 particles and with open boundary conditions.

figure $4(\mathrm{c})$ (stars). The second transition at a larger value of $\beta$ corresponds to the ordering of mn native contacts, and is represented by the second, sharp peak of $\Delta_{\eta}^{\text {next }}$ in figure 4 (c) (stars).

In order to rationalize the results from our MC simulation we have derived the phase diagram of our system within the mean field approximation. Technically this is achieved by mapping our four-species model onto a spin model with two coupled Ising variables $(\sigma, \tau)$ per site. This mapping is given by $\mathrm{A} \rightarrow(1,1), \mathrm{B} \rightarrow(1,-1), \mathrm{C} \rightarrow(-1,1)$ and $\mathrm{D} \rightarrow(-1,1)$. The single site probability in terms of the Ising spins is given by

$$
P_{i}(\sigma, \tau)=\frac{1}{4}\left(1+m_{i, 1} \sigma+m_{i, 2} \sigma \tau \div m_{i, 3} \tau\right)
$$

The generalized single site 'magnetizations' defined by the vectors of coefficients $\mathbf{m}_{i}=$ $\left(m_{i, 1}, m_{i, 2}, m_{i, 3}\right)$ identify the order parameters of our system. Without dwelling on the details here, we can formulate the mean field free energy $\mathcal{F}\left[\left(\mathbf{m}_{i}\right)\right]$ of the system as a functional of the 


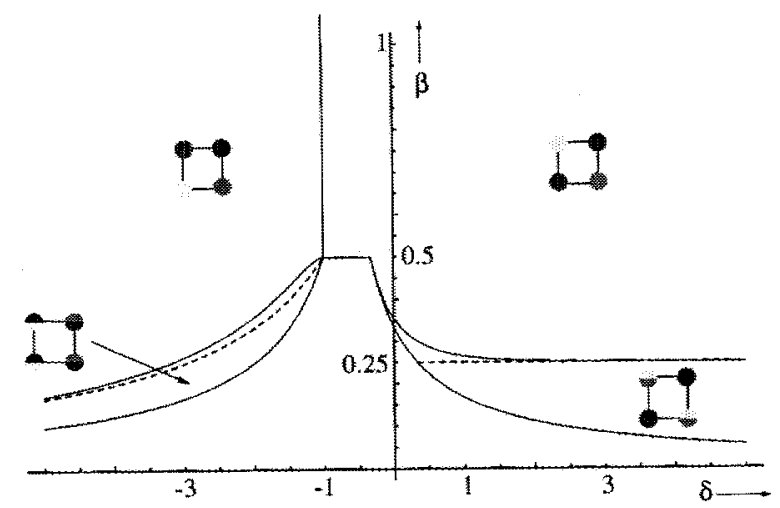

Figure 6. Computed mean-field phase diagran for the fozr-component colloidal system: the inverse transition temperature, $\beta \equiv \epsilon / k_{\mathrm{B}} T$, versus the strength of competitive binding, $\delta$ (see main text for the detaited explanation).

single site magnetizations. Minimizing this functional yields the equilibrium phase structure. More specifically, the phase boundares can be determined analytically for transitions from the high temperature disordered state or almost analytically for transitions from pre-ordered states using a bifurcation analysis of the minimization equations. The resulting phase diagram is shown in figure 6 . We find that this analysis essentially reproduces all the salient features observed in the simulations. For $\delta \geqslant-\frac{1}{3}$ we expect two consecutive transitions, the first to a state in which the two sets of nnn compatible pairs of particles separate onto two sublattices in a chequerboard fashion, followed by a transition in which each of the individual species preferentially occupies its position within the structure of the design goal, which is the predicted ground state. For $-1 \leqslant \delta \leqslant-\frac{1}{3}$, we expect a single transition into a state which immediately possesses the symmetry of the design goal structure. For $\delta \leqslant-1$ the ground state of the system is no longer the design goal structure, but rather a striped or columnar phase, in which the individual columns are ordered, but there is no correlation between the ordering on neighbouring columns. Note that in this whole region we also expect a pre-ordering transition towards a phase in which the columnar pattern first establishes itself, with pairs of species dividing themselves among the alternating columns, but lacking order within the columns. In the simulations performed at $\delta=-1$ (curve (e) figure 3) we have not resolved this pre-ordering transition, but do observe a very broad specific heat peak, which is certainly consistent with the prediction. Clearly, there are discrepancies in the predictions of the values of the transition temperatures between the theory and the simulation. However, one cannot expect the mean field approximation to account for all the quantitative features of the phase diagram in this naturally strongly fuctuating $2 \mathrm{D}$ system.

\subsection{Varying range and strength of interactions in DNA-coated colloidal system}

We now turn to the question of how one could practically vary the range and the strength of s\$DNA-linker-induced interactions in the system of DNA-coated colloids. Specifically we would like to show how can one realize experimentally the model interaction network investigated above. In this context the importance of competitive binding, as introduced in the previous section, will become apparent. 


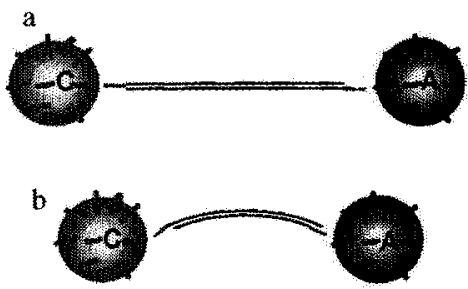

\begin{abstract}
Figure 7. Schematic representation of the scenario whete a linker designed to provide the binding between a colloid pair separated by a specific distance (say nnn separation) (a), also provides the binding at a smaller distance (say nn separation) for the same colloid pair (b), bat with a higher energy of the bond as compared to (a). See also figure 2. The bond energy in this case is the DNA binding energy plus the bending energy. Practically the linker can be a dsDNA molecule with the length of the order of $50 \mathrm{~nm}$ with ssDNA 'sticky', tecognition ends.
\end{abstract}

In the case of ssDNA-coated colloids, the strength and the characteristic range of the interparticle interaction potential are determined by the surface grafting densities and sequences of the probe sSDNAs on colloids, and by the concentration of the complementary ssDNA linkers. The properties of these linkers depend on their length and flexibility. In addition, the interaction depends on the properties of the solvent [3-7]. These effective, DNA-linker-induced potentials were studied theoretically in a number of recent works [21-24].

Our main observation is that the required pattern of interaction can be realized using ssDNA-coated nanoparticles connected by ssDNA linkers of varying lengths. In particular, in order to self-assemble a four-component system with a ground state as shown in figure 2 , one can use linkers of two different spacings: shorter $A^{\prime} B^{\prime}, B^{\prime} C^{\prime}, C^{\prime} D^{\prime}$, and $A^{\prime} D^{\prime}$ linkers with the bond length equal to the nearest neighbour lattice spacing. This is in combination with longer $\mathrm{A}^{\prime} \mathrm{C}^{\prime}$ and $\mathrm{B}^{\prime} \mathrm{D}^{\prime}$ linkers which favour bonds with the next-nearest neighbour lattice spacing, figure 2(a).

This design rule imposes the ground-state stripe structure shown in figue 2 . The important factor in the experimental realization of this strategy is the fact that the interactions that are 'programmed" to act between next-nearest neighbours (e.g. negative $K_{\mathrm{AC}}$ achieved using $\mathrm{A}^{\prime} \mathrm{C}^{\prime}$ linkers) will also compete for the nearest neighbour AC colloid pairs, due to the flexibility of DNA bonds (the DNA persistence length is $50 \mathrm{~nm}$ ). Therefore, the corresponding competitive interactions ( $J_{\mathrm{AC}}$ in this case) cannot be switched off entirely (see schematic plot, figure 7 and also figure 1).

For example, simple, lattice models (see e.g. $[23,24]$ ) predict that the linker-induced potentials $J_{\alpha \beta} \sim-k_{\mathrm{B}} T M \ln \left(1+z_{\alpha \beta}\right)$ and $K_{\alpha \beta} \sim-k_{\mathrm{B}} T M \ln \left(1+z_{\alpha \beta}^{\text {next }}\right)$, where $M$ is the maximal possible number of DNA bonds between neighbouring colloids $\alpha$ and $\beta$, and $z_{\alpha \beta}=\exp \left[\left(\mu_{\alpha \beta}-\varepsilon_{\alpha \beta}\right) / k_{\mathrm{B}} T\right], z_{\alpha \beta}^{\text {next }}=\exp \left[\left(\mu_{\alpha \beta}-\varepsilon_{\alpha \beta}^{\text {next }}\right) / k_{\mathrm{B}} T\right]$, with $\mu_{\alpha \beta}$ being the chemical potential of $\alpha^{\prime} \beta^{\prime}$ DNA linkers in solution, and $\varepsilon_{\alpha \beta}$ and $\varepsilon_{\alpha \beta}^{\text {nex€ }}$ is the energy of the bond for $n$ and nnn colloid $\alpha \beta$ pairs, respectively. For the situation shown in figure 7 , clearly, $\varepsilon_{\mathrm{AC}}^{\mathrm{next}}<\varepsilon_{\mathrm{AC}}$, and this corresponds to the scenario of weak competitive binding. In general, $\varepsilon_{\alpha \beta}$ sensitively depends on the linker mechanical properties, and inter-particle separation.

If linkers have spacers composed of e.g. dSDNA molecules (see figure 7), the typical length of linkers providing weak competitive binding is restricted to about the dsDNA persistence length of $50 \mathrm{~nm}$. If however spacers are composed of carbon nanotubes with DNA recognition ends [18], linkers can be stiff within the range of the order of hundreds of nanometres. If, for example, linkers are very long and flexible (e.g. dsDNA molecules which are much longer than $50 \mathrm{~nm}$ ), the optimal inter-particle spacing is determined by the dsDNA radius of gyration. 


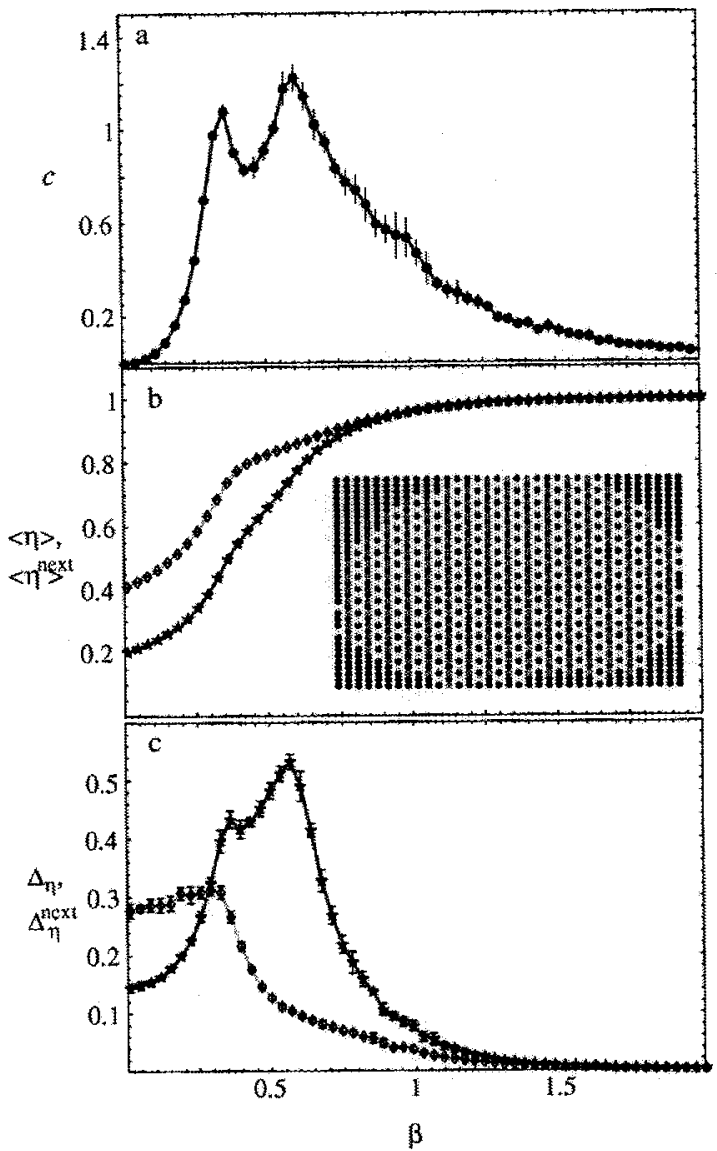

Figure 8. Computed thermodynamic and structural properties of the four-component model with yacancies in the case of weak competitive binding; speciftc heat $c$ per particle (a); mean value of the order parameter $\left\{\eta\right.$ ) in (b); and fuctuation of the order parameter $\Delta_{\eta}$ in (c). In (b) and (c) the two curves with diamonds and stars correspond to the nearest neighbour $\mathrm{AB}$ and the next-nearest neighbour $\mathrm{AC}$ particle pairs, respectively (see main text and figure 2). The interaction network for particles used to compate these plots is identical to the one used to compate figure 3(a), where $\delta=+1$. The energies of vacancies (V) are all set to be zero: $J_{\Lambda \mathrm{V}}=J_{\mathrm{BV}}=J_{\mathrm{CV}}=J_{\mathrm{VV}}=0$, $K_{\mathrm{AV}}=K_{\mathrm{BV}}=K_{\mathrm{CV}}=K_{\mathrm{DV}}=0$, and $J_{\mathrm{VV}}=K_{\mathrm{VV}}=0$. The inset in (b) shows a typical simulation snapshot of the system at low temperatures (high $\beta$ ). Vacancies are coloured in black. MC simulations were performed with 1225 particles and with open boundary conditions. The concentration of all the species (four species of colloids and vacancies) was chosen to be $20 \%$

\section{Kinetics of ordering}

In the previous section we have addressed the importance and the realizability of the competitive bindings in our design problem. In a sense these competitive interactions provide a degree of freedom one can explott to optimize the process of achieving the design goal structure. Specifically, the results of section 2 show that for a wide range of values of $\delta>-1$ the design goal structure is the predicted stable ground state. However, an additional requirement is that this state should be kinetically accessible, assuming that one starts from a high temperature 


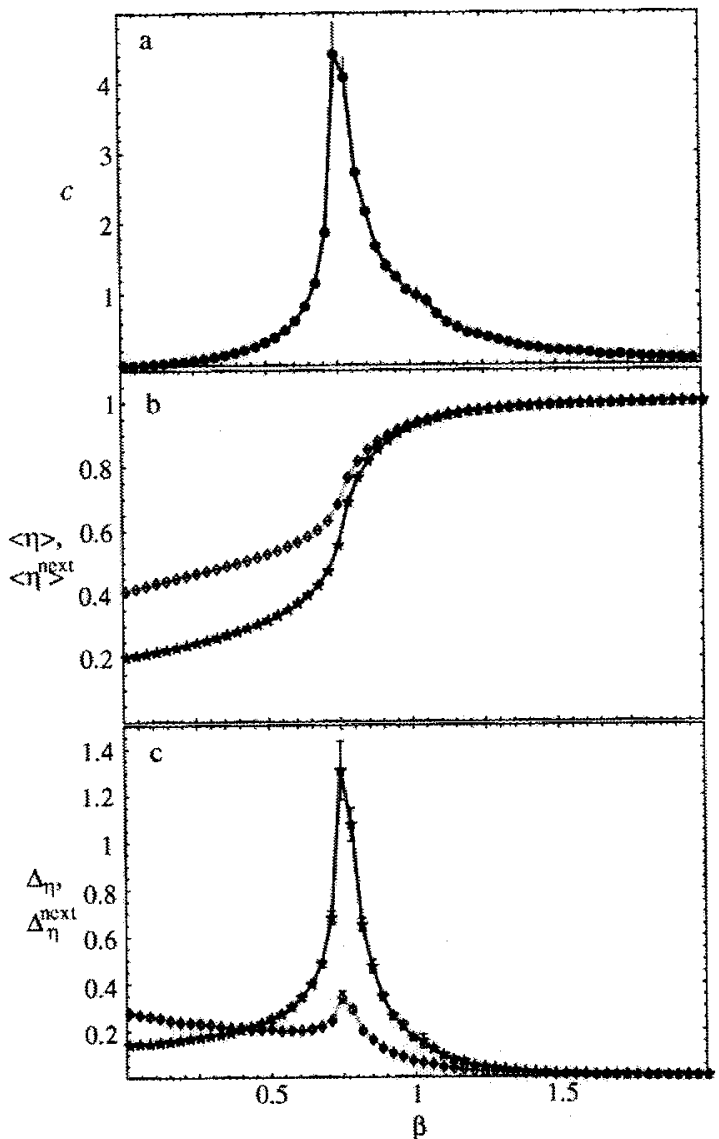

Figure 9. Computed thermodynamic and structural properties of the four-component model with vacancies in the case of strong competitive binding: specific heat $c$ per particle (a); mean value of the order parameter $(n)$ in $(b)$; and fuctwation of the order parameter $\Delta_{\eta}$ in $(c)$. In (b) and (c) the two curves with diamonds and stars correspond to the nearest neighbour $\mathrm{AB}$ and the next-nearest neighbour $\mathrm{AC}$ particle pairs, respectively (see main text and figure 2). The interaction network for particles ased to compute these plots is identical to the one used to compute figure $3(\mathrm{c})$, where $\delta=-0.5$. The energies of vacancies (V) are all set to be zero: $J_{A V}=J_{B V}=J_{C V}=J_{D V}=0$, $K_{A V}=K_{B V}=K_{C V}=K_{D V}=0$, and $J_{\mathrm{VV}}=K_{\mathrm{VV}}=0$. MC simulations were performed with 1225 particles and with open bondary conditions. The concentration of all the species (fout species of collotds and vacancies) was chosen to be $20 \%$

or low concentration initial state and subsequently tries to quench the system into the ordered state. We therefore define the optimally designed system as the one in which the kinetics of ordering is fastest.

Our key observation now is that the differences in the thermodynamic and structural behaviour of the model DNA-linked colloidal systems analysed in the previous section and shown in figutes 4 and 5 have an important implication on the kinetics of ordering.

To illustrate this point we look at the thermodynamic and structural properties of our lattice model but now with vacancies, in order to allow for more realistic vacancy mediated local rearrangements of particles, rather than the strongly correlated particle exchange moves 


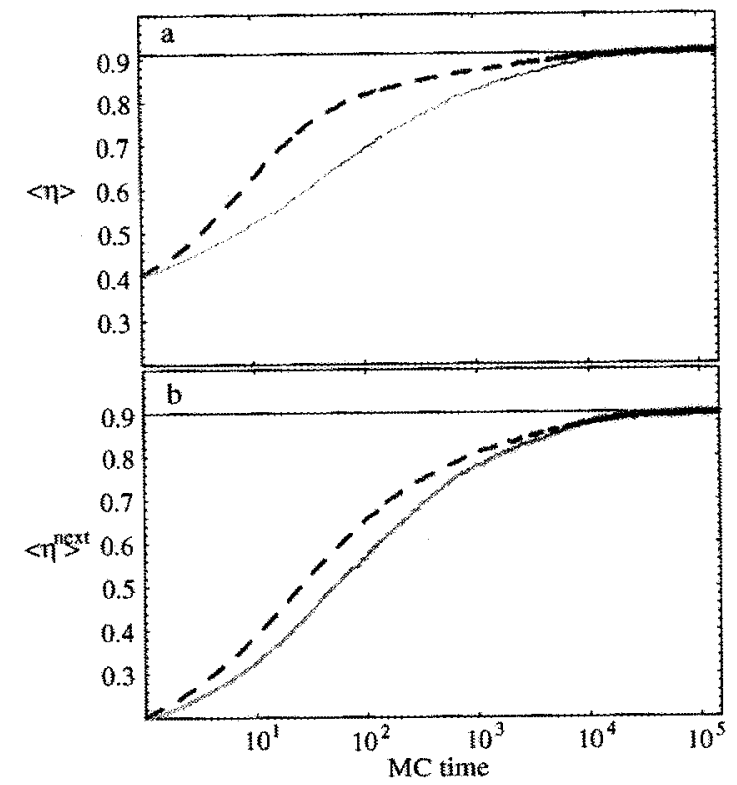

Figure 10. Computed time dependence of the order parameter relaxation from the random configuration to the equilibrium state upon instantaneous quenching. This is computed for the four-component system of colloids with vacancies. Plot $(a)$ represents the relaxation of $\langle\eta\rangle$ for the nn collote pairs (fraction of nn pairs $A B$ ), and (b) represents the relaxation of $\left\langle\eta^{\text {next }}\right\rangle$ for mn colloid pairs (fraction of mn pairs $A C$ ). The dashed and solid carves on each plot correspond to $\delta=+1$ and $\delta=-0.5$, respectively. The values of $\beta \equiv \beta^{*}$ at which the instantaneous quench is performed are $\beta^{*} \simeq 0.794$ for $\delta=+1$, and $\beta^{*} \approx 0.957$ for $\delta=-0.5$. These values of $\beta^{*}$ are chosen in such a way that the equilibrium values of $\langle\eta\rangle^{*} \approx 0.9$ and $\left\langle\eta^{\text {next }}\right\rangle^{*} \approx 0.91$ for both cases with $\delta=+1$ (see figure $8(\mathrm{~b})$ ) and $\delta=-0.5$ (see figure $9(\mathrm{~b})$ ). These threshold, equilibrium values of the order parameters are marked by the horizontal lines in (a) and (b). Each of the four curves is the average over $70 \mathrm{MC}$ trajectories.

that the vacancy-less model requires. We again compute the specific heat $c$, the mean value of the order parameter $(\eta)$ and the fluctuation of the order parameter $\Delta_{\eta}$ for the four-component system with vacancies. The energy of this system is analogous to equation (1), except for the presence of the fifth 'species' $V$-the vacancies, but for simplicity we assume that the vacancies do not interact with the particles of all other species $\left(J_{\mathrm{vs}}=K_{\mathrm{Vs}}=0\right)$.

The results of these simulations are shown in figures 8 and 9 , and they are similar to the results obtained in the case without vacancies and shown in figures 4 and 5 , respectively. These two cases correspond to the situation of weak (figure 8) and strong (figure 9) competitive binding. In each case with vacancies present, the order-disorder transition temperature shifts to a lower value (higher value of $\beta$ ) as compared with the corresponding case without vacancies. In the case with vacancies the order-disorder transition is also slightly more smeared out as compared with the case without vacancies.

The relaxation to equilibrium behaviour of the order parameters $\langle\eta\rangle$ and $\left\langle\eta^{\text {next }}\right\rangle$ for $\mathrm{m}$ (AB) and nnn ( $A C$ ) colloid pairs, respectively, is shown in figure 10. This is computed upon the instantaneous quench from random configurations of colloids to the equilibrium state at a specific value of $\beta=\beta^{*}$. The dashed and solid curves on each plot correspond to $\delta=+1$ and $\delta=-0.5$, respectively. The values of $\beta \equiv \beta^{*}$ at which the instantaneous quench is performed are $\beta^{*} \simeq 0.794$ for $\delta=+1$, and $\beta^{*} \simeq 0.957$ for $\delta=-0.5$. These values of $\beta^{*}$ are chosen in 


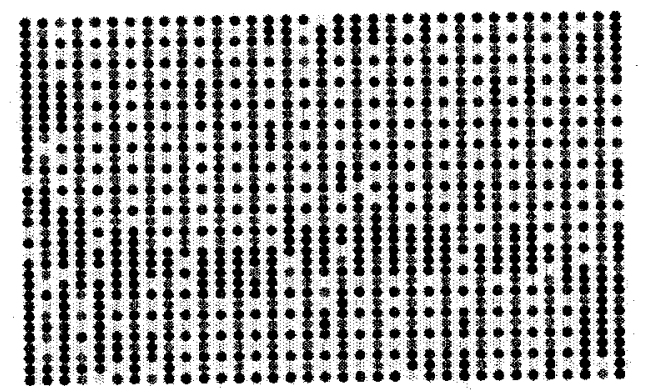

Figure 11. Typical snapshot of the equilibrated system at $\left\langle\eta_{3}\right\rangle^{*} \simeq 0.9$ and $\left\langle\eta^{\text {next }}\right)^{*} \simeq 0.91$. Vacancies are coloured in black.

such in way that the equilibrium values of $\langle\eta\rangle^{*} \simeq 0.9$ and $\left\langle\eta^{\text {next }}\right\rangle^{*} \simeq 0.91$ for both cases with $\delta=+1$ (see figure $8(\mathrm{~b})$ ) and $\delta=-0.5$ (see figure $9(\mathrm{~b})$ ). These threshold values of the order parameters are marked by the horizontal lines in figures $10(\mathrm{a})$ and (b). A typical snapshot of the equilibrated system with $\langle\eta\rangle \simeq 0.9$ and $\left\langle\eta^{\text {next }}\right\rangle \simeq 0.91$ is shown in figure 11 .

We observe that in the case of weak competitive binding, $\delta=+1$, the system relaxes faster to the equilibrium, ordered state as compared with the case of strong competitive binding, $\delta=-0.5$. We stress the fact that the thermodynamic transition at $\delta=+1$ is less cooperative than at $\delta=-0.5$ (compare figure 8 with figure 9); however, the kinetics of ordering is faster at $\delta=+1$. Pre-ordering of the nn 'native' contacts at $\delta=+1$ (characterized by the first peak in $c$ and $\Delta_{7}$ ) speeds up the entire ordering of the system. This suggests that the optimal design strategy should not just aim to reduce the strength of competitive binding, but also to make the ordering transition structurally 'staged'. Such a 'staged' ordering would facilitate the kinetics due to the formation of pre-ordered superstructures, which in their turn facilitate the ordering of the rest of the system.

\section{Summary and conclusion}

In summary, we propose that non-spherically symmetric, ordered structures can be designed using spherically symmetric DNA-coated nanoparticles and DNA linkers of non-equal lengths which form bonds of different spacings. We considered an example of the design of the stripe structure in the four-component system of colloids with spherically symmetric interaction potentials. We varied the strength of competitive binding in the designed stripe structure and monitored the thermodynamic, structural and kinetic properties of the system. In all cases the system was imposed to have exactly the same ground state energy and the highest degree of connectivity of particles in the ground state. We established that when the strength of competitive binding is weak, the four-component system exhibits two consecutive ordering transitions. These transitions arise due to the 'staged' ordering of superstructures within the system. Most importantly, we predict that these structural, "staged' ordering facilitates the kinetics of colloidal ordering. This is in spite of the fact that the cooperativity of the order-disorder transition is not the highest when the strength of competitive binding is the weakest. Based on these observations, we propose that the optimal design strategy should not only provide the weakest competitive binding, but should also aim at the 'staged' ordering of superstructures in the multi-component system.

\section{Acknowledgments}

DL is grateful to D Fuks, C Mirkin, T Schmatko, and A Vaskevich for useful discussions, and to $B$ Bozorgui and I Coluzza for critical reading of the manuscript. This work is part of the 
research program of the Stichting voor Fundamenteet Onderzoek der Materie (FOM), which is financially supported by the Nederlandse organisatic voor Wetenschappelijk Onderzoek (NWO).

\section{References}

(1) Katz E and Wilfner I 2004 Angew, Chem. Int. Edn 436042

[2] Seeman N C 2003 Nalure 421427

[3] Mirkin C A, Letsinger R L, Mucic R C and Storhoff J J 1996 Nature 382607

[4] Taton T A, Mrkin C A and Letsinger RL 2000 Science 2891757

[5] Jin R, We G, Li Z, Mitkin C A and Schatz G C 2003 J. Am. Chem. Soc 1251643

[6] Biancaniello P L, Kim A J and Crocker J C 2005 Phyx. Rev. Lett. 94058302

[7] Valignat MP. Theodoly O, Cocker JC, Russel W B and Chaikin P M 2005 Proc. Natl Acad. Sci. USA 1024225

[8] Soto C M, Srinivasan A and Ratna B R 2002 J. Asu. Chem. Soc. 1248508

[9] Kiang C H 2003 Physica A 321,64

[10] Sun Y, Harris N C and Klang C H 2005 Physica A 35089

[11] Harris N C and Kiang C H 2005 Phys. Rev. Lett. 9504610

[12] Rogers P H, Michel E, Bauer C A, Vanderet $S$, Hansen D, Roberts B K, Calvez A, Crews J B, Lau K O, Wood A, Pine D I and Schwarte P V 2005 Langmuir 215562

[13] Le I D, Pinto Y, Seeman N C, Musier-Forsyth K, Taton T A and Kiehl R A 2004 Nono Leth. 42343

114] Seeman N C and Lukeman P S 2005 Rep. Prog. Phys. 68237

[15] Zhang Z L and Glozer S C 2004 Nano Lett 4 1407

[16] Mokari T, Rothenberg B, Popov I, Costi R and Banin U 2004 Science 3041787

[17] Caswell K K, Wilson J N, Bunz U H F and Muphy C J 2003 J. Am. Chem. Soc. 12513914

[18] Willams K A, Veenhuizen P T M, de la Tore B G, Eritja R and Dekker C 2002 Nature 420761

[19] Keren K, Berman R S, Buchstab E, Sivan U and Bratn E 2003 Science 3021380 Keren K, Krueger M, Gilad R, Ben-Yoseph G, Sivan U and Bram E 2002 Science 29772

[20] Ye Y H, Mayer T S, Khoo I C, Divliansky I B, Abrams N and Mallouk T E 2002 J. Mater. Chem. 123637

[21] Tkachenko A V 2002 Phys. Rev. Lett. 89148303

[22] Licata N A and TKacherko A V 2005 Preprint cond-13at 0504407

[23] Lukatsky D B and Frenkel D 2004 Phyx. Rev, Lett. 92068302

[24] Lukatsky D B and Frenkel D 2005 1. Chem. Phys, 122214904

[25] Yamanura Y, Moriyana S, Tsuji T, Iwasa Y, Koyano M, Katayama S and to M 2004 J. Alloys Compounds $\mathbf{3 8 3} 338$ 